\title{
A New Actuation Approach for Human Friendly Robot Design
}

\author{
Zinn, M. ${ }^{2}$, Khatib, O. ${ }^{1}$, Roth, B. ${ }^{2}$, and Salisbury, J.K. ${ }^{1}$ \\ 1 Robotics Laboratory, Department of Computer Science \\ 2 Design Division, Department of Mechanical Engineering \\ Stanford University, Stanford, California 94305
}

\begin{abstract}
In recent years, many successful robotic manipulator designs have been introduced. However, there remains the challenge of designing a manipulator that possesses the inherent safety characteristics necessary for human-friendly robotics. In this paper, we present a new actuation approach that has the requisite characteristics for inherent safety while maintaining the performance expected of modern designs. By drastically reducing the effective impedance of the manipulator we show that uncontrolled impact loads can be reduced by an order of magnitude or more, as compared to conventional manipulator designs. A discussion of the actuation topology is presented along with analytical and experimental results validating the efficacy of our approach.
\end{abstract}

\section{Introduction}

One of the major issues in introducing robots into human environments is safety. Without a high degree of confidence in their inherent safety, robotic manipulators will never be accepted for use in close proximity to humans. However, safety alone will not guarantee the success of human friendly robotics. These robotic manipulators must also possess a level of performance that is expected of modern robotic manipulators.

Inherent safety is achieved through the use of multiple strategies, involving all aspects of manipulator design including the mechanical, electrical, and software architectures. However, the biggest danger present when working in close proximity with robotic manipulators is the potential for large impact loads which can result in serious injury or death. To evaluate the potential for serious injury due to impact we can use the HIC index, an empirical formula developed by the automotive industry to correlate head acceleration to injury severity. A simple two degree of freedom mass-spring model can be used to evaluate predicted head accelerations, a ( $\mathrm{t}$ ) expressed in g's, and the resulting HIC index(Equation 1).

$$
H I C_{\Delta T}=\left(t_{2}-t_{1}\right)\left[\frac{1}{\left(t_{2}-t_{1}\right)} \int_{t_{1}}^{t_{2}} a(t) d t\right]^{2.5} \quad \Delta T=t_{2}-t_{1}=15 \mathrm{~ms}
$$


For the PUMA 560, an impact at $1 \mathrm{~m} / \mathrm{s}$ velocity produces a maximum HIC index more than enough to cause injury ${ }^{1}$ (see Figure 1)

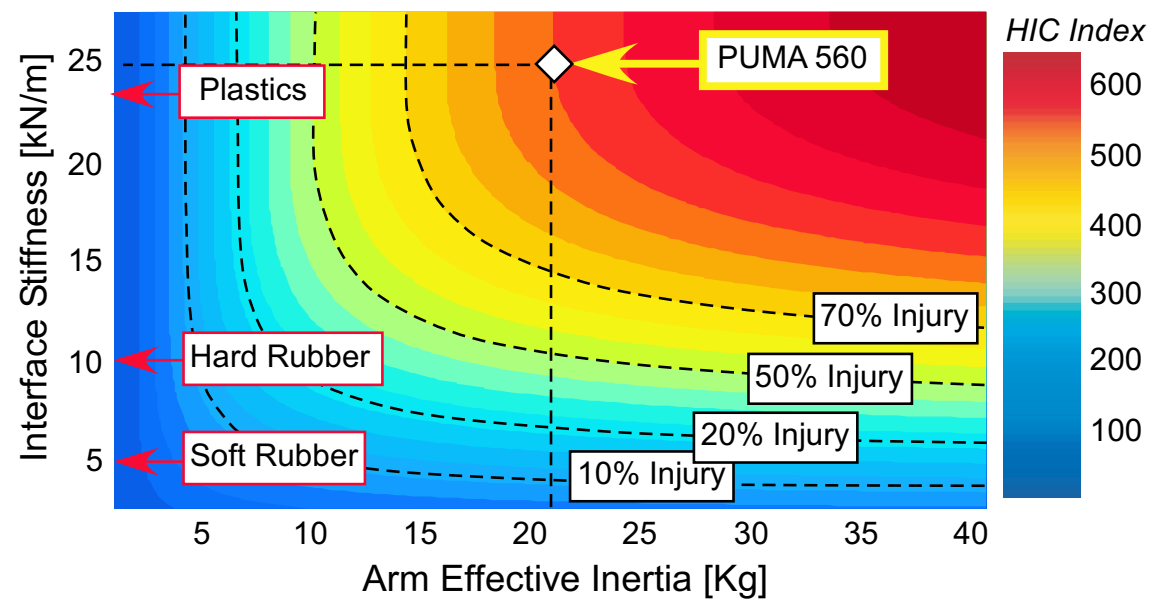

Fig. 1. Head injury criteria as a function of effective inertia and interface stiffness

The addition of a compliant covering can reduce impact loading by an order of magnitude or more. However, the amount of compliant material required to reduce impact loads to a safe level can be substantial ${ }^{2}$. Clearly, this does not address the root cause of high impact loads - namely the large effective inertia of most modern robotic arms.

Previous attempts to build lightweight, low inertia manipulators have been met with limited success. Due to the flexibility of cable transmissions, control bandwidths are limited. The non-collocated nature of the remotely located actuators and flexible transmission limits the tasks that can be achieved to those that require torques whose frequencies lie below the fundamental mode, which can be as low as $5 \mathrm{~Hz}$. Other approaches [1], attempt to solve these problems with the use of high performance cable transmissions and light, stiff structural materials. This design approach prevents interaction with the robot control system by increasing the structural modes to frequencies above the control bandwidth. The need for high performance components and materials means that the cost of such systems is high. In addition, the stiff connection between the manipulator link and the actuator couples

\footnotetext{
$\overline{1}$ The HIC index is correlated with the Maximum Abbreviated Injury Scale (MAIS) to provide a mapping from the calculated HIC values to the likelihood of an occurrence of a specific injury severity level. In Figure 1, HIC values and the corresponding likelihood of a concussive injury (or greater) are shown

${ }^{2}$ For the PUMA robot, the thickness of a compliant cover required is more than 5 inches, assuming an impact velocity of $1 \mathrm{~m} / \mathrm{s}$ and an allowable maximum HIC index of 100
} 
their inertias. The increase in the effective link inertia can be substantial considering the $\mathrm{N}^{2}$ amplification of the actuators inertia through the transmission. The most promising manipulator designs to date have utilized the joint torque control approach [2]. Perhaps the most successful of these has been the new DLR lightweight arm design [3]. The implementation of joint torque control allows for near zero low frequency impedance, which gives the DLR arm excellent force control characteristics. However, above the control bandwidth, joint torque control is ineffective at reducing the impedance of the manipulator. The open loop characteristics of the manipulator and reflected actuator inertia dominate. Thus, the magnitude of impact loads, which are determined by the high frequency impedance of the contacting surfaces, are not attenuated.

\section{New Actuation Approach: Distributed Elastically Coupled Macro Mini Actuation}

To address this challenge, we propose a new approach that seeks to relocate the major source of actuation effort from the joint to the base of the manipulator. This can substantially reduce the reflected inertia of the overall manipulator. Performance is maintained with small actuators collocated with the joints. Our approach divides the torque generation into low and high frequency components and distributes these components to the arm location where they are most effective. We call this approach Distributed Elastically Coupled Macro Mini Actuation (DECMMA).

The proposed approach is analogous to the design of robotic manipulators for use in zero gravity. Under such conditions, gravity induced torques do not exist. Joint actuators provide torques related only to the task, such as trajectory tracking and disturbance rejection, both of which are primarily medium to high frequency in content. We achieve the zero gravity analogy by compensating for low frequency torques using the low frequency actuators located at the base of the manipulator. With the effects of gravity and low frequency torques compensated, joint torque requirements become similar to those encountered by a zero gravity robotic manipulator.

The efficacy of this approach can be seen clearly when one considers that most manipulation tasks involve position or force control which are dominated by low frequency trajectory tracking or DC load torques. High frequency torques are almost exclusively used for disturbance rejection. Even haptic device torque profiles, which might require rapid changes approximating a square wave input, have a torque magnitude versus frequency curve that falls off with increasing frequency by $1 / \omega$ (see Figure 2). This torque versus frequency profile is ideally fit using a large output, low frequency actuator coupled with a high frequency servomotor.

In contrast to early efforts at coupled actuation [4], the low frequency torque actuator is located remotely from the actuated joint. This is partic- 

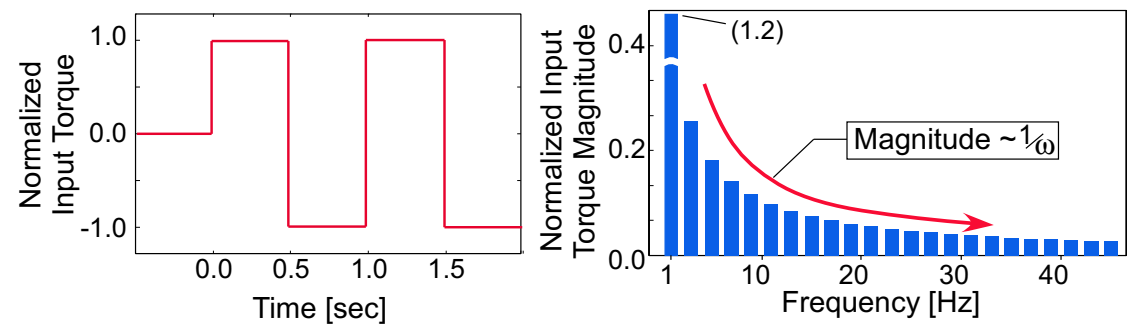

Fig. 2. Torque vs frequency: $1 \mathrm{~Hz}$ square wave

ularly advantageous as the low frequency components of most manipulation tasks are considerably larger in magnitude than the high frequency components and consequently require a relatively large actuator. Locating this large actuator at the base significantly reduces the weight and inertia of the manipulator

The high frequency actuators are located at the manipulator joints and connected through a stiff, low friction transmission, providing the high frequency torque components that the low frequency base actuators cannot. The high frequency torque actuator must be connected to the joint inertia through a connection, which produces a high primary mode vibration frequency. By locating the actuator at the joint and by using a low inertia servomotor we can achieve this high bandwidth connection with a minimum amount of weight and complexity.

\subsection{DECMMA Actuation}

In order for the DECMMA approach to work properly, both the high and low frequency actuators must have zero or near zero impedance. This is due to the fact that during power transfer the actuator torques will add nondestructively only if their respective impedance is zero. In particular, each actuator must not have significant impedance within the frequency range of the opposing actuator. Only if this condition is true will the DECMMA concept work.

For the high frequency actuation, very low impedance is achieved by using a low inertia servo motor connected to the manipulator through a low friction, low reduction cable transmission. The reduced torque output that results from the use of a low reduction transmission is balanced against the reduced reflected inertia and motor friction and represents one of the design trades of the DECMMA concept. Unfortunately, this approach can not be applied to the low frequency base actuation. The large torques required to react gravity loads make it impossible to achieve low reflected impedance without employing very high performance actuators, most of whose power and performance would be under utilized. To achieve the near zero impedance required 
we use a new type of actuator topology, referred to as Series Elastic Actuation (SEA) [5] that was developed specifically to address the problems of high impedance actuators. The SEA actuator topology maintains the high power and torque density of a high ratio geared DC torque motors while also providing the very low impedance required for the DECMMA approach. The penalty paid in implementing the SEA approach is a significant reduction in the high frequency torque capability of the actuator. However, the DECMMA approach does not require that the base actuator be capable of supplying high frequency torques and thus this limitation is an acceptable trade off. While details of SEA are contained in $[5,6]$ a brief overview of the concept and its implications for the DECMMA approach is given below.

\subsection{Low Frequency Actuation: Series Elastic Actuation}

The SEA approach seeks to mitigate the limitations of conventional gearhead actuators, namely the high impedance associated with the reflected inertia and friction, by placing an elastic element between the output of the actuator and the robotic link. The elastic element limits the high frequency impedance of the actuator to the stiffness of the elastic coupling. To limit the low frequency impedance, and thus transform the actuator into an approximate pure torque source, a linear feedback system is implemented to regulate the output torque of the actuator-spring system. (See Figure 3).

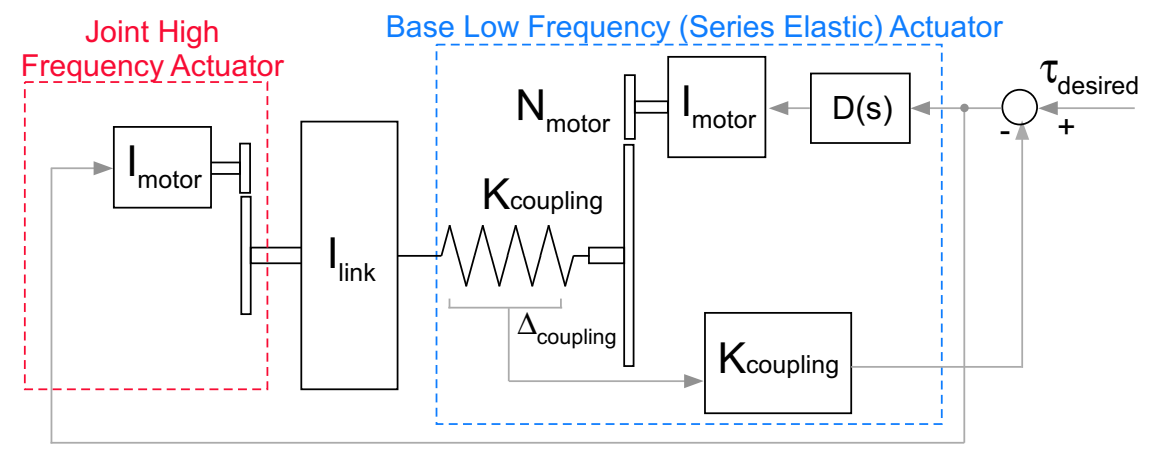

Fig. 3. Distributed elastically coupled macro mini actuation topology

The main advantage of the SEA topology is that it provides low output impedance across the frequency spectrum. As shown in $[5,6]$, the SEA topology reduces the output impedance of the SEA actuator in proportion with the stiffness of the elastic coupling (Equation 2). At frequencies below the closed loop bandwidth of the SEA controller, the output impedance is reduced as a function of the control gains. Impedance reduction of 10x-100x is common and is only limited by the maximum obtainable bandwidth. At 
frequencies above the closed loop bandwidth, the output impedance reduces to the stiffness of the elastic coupling.

$$
\frac{F(s)}{X(s)}=\quad \frac{s^{2}\left(N_{\text {motor }}\right)^{2} I_{\text {motor }}}{\frac{s^{2}\left(N_{\text {motor }}\right)^{2} I_{\text {motor }}}{K_{\text {coupling }}}+1+N_{\text {motor }} D(s)}
$$

This is in contrast to other approaches, such as joint torque control [7], which have good low frequency impedance but suffer from large high frequency impedance. This is particularly problematic as impact loads are largely affected by the high frequency impedance of the impacting surfaces.

There are trade offs with using the SEA actuators. Due to velocity and torque saturation of the SEA actuator, the maximum output torque above the open loop mode of the system ${ }^{3}$ falls off as $1 / \omega$ regardless of the control loop controller bandwidth [6]. This behavior is an open loop characteristic of the SEA actuator topology and represents a fundamental physical limitation of the actuator. The choice of the elastic coupling stiffness (in relation to the manipulator and motor reflected inertia) determines the open loop mode frequency. A stiffer coupling improves the high frequency open loop torque performance but adversely affects the desirable closed and open loop impedance characteristics. This design trade represents the main challenge when implementing an SEA actuator.

\section{Control Approach}

The DECMMA control approach seeks to exploit the distributed-parallel actuation's unique characteristics to construct a near perfect torque source. The characteristics of a perfect torque source, consisting of zero output impedance and infinite control bandwidth, would enable a manipulator to possess the characteristics necessary for both inherent safety and high performance tasks. While a perfect torque source is impossible to achieve, a near perfect torque source, with low output impedance relative to the driving load and high bandwidth torque capability offers much of the same advantages.

The control structure shown in Figure 4(A) utilizes the low frequency base actuator's low pass filter characteristics to partition the control torques into low and high frequency components. By using the actual measured torque output from the low frequency base actuators in combination with the desired torque, we automatically compensate for the non-ideal behavior of the base actuators. Assuming that the smaller joint actuators can produce this torque, the combined torque sum is a perfect realization of the desired torque. The frequency partitioning can be clearly seen if we rearrange the structure in Figure 4(A) into a pure parallel structure, as shown in Figure 4(B). As seen in Figure 4(B), the equivalent base actuator falls off at high frequency while the

\footnotetext{
${ }^{3}$ SEA open loop mode: unforced coupled motion of actuator and manipulator link inertias through the compliant coupling
} 


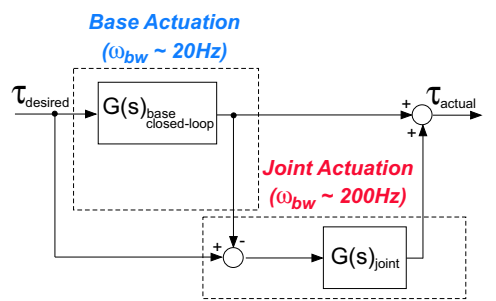

(A)

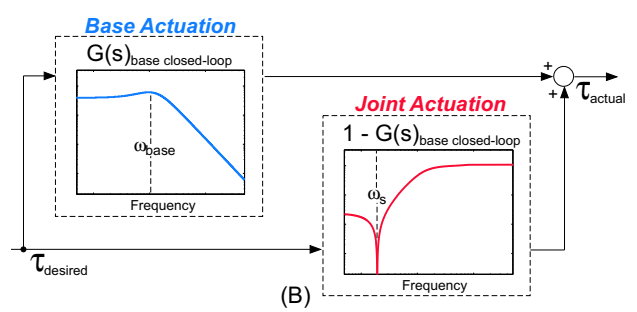

Fig. 4. (A) DECMMA control structure (B) Equivalent parallel structure

equivalent joint actuator approximates a double lead filter, which adds phase to combined system and attenuates the DC and low frequency components commanded to the high frequency actuator. The combined actuator control

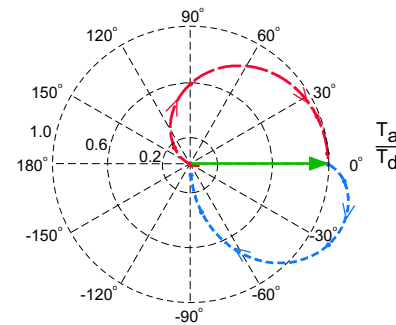

(A)

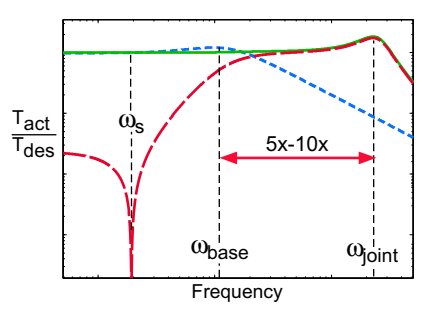

(B)

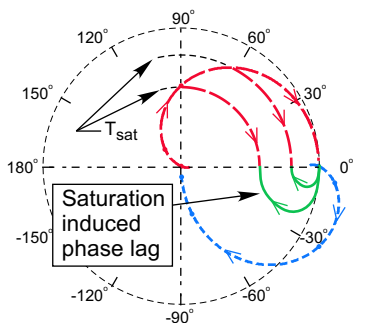

(C)

$$
----\mathrm{G}(\mathrm{s})_{\text {base }}--\mathrm{G}(\mathrm{s})_{\text {joint }}-\mathrm{G}(\mathrm{s})_{\text {DECMMA }}
$$

Fig. 5. (A) Perfect torque source: Base, joint, and combined DECMMA actuator torque magnitude vs phase polar plot (B) Near perfect torque source: Base, joint, and combined DECMMA actuator torque magnitude vs frequency $(\mathrm{C})$ Breakdown of perfect torque source due to saturation: Base, joint, and combined DECMMA actuator torque magnitude vs phase polar plot

structure creates a perfect torque source (in the linear sense where the torques sum to unity magnitude and zero phase), up to the first resonance mode frequency $\left(\omega_{\text {joint }}\right)$ as seen in Figure $5(\mathrm{~A})(\mathrm{B})$.

The combined parallel system's saturation torque is determined by the saturation torque profile of both the low and high frequency actuators. Parallel system torque saturation represents the threshold above which the joint actuator can no longer compensate for the phase and magnitude error of the low frequency base actuator. Commanded torques which force the high frequency joint actuator to saturate will cause both magnitude errors and phase lag to occur, invalidating the perfect torque source characteristics of the combined parallel actuation, and limiting the achievable task bandwidth (see Figure $4(\mathrm{C})$ ). The sizing of the low frequency base and high frequency joint actuators, as well as selection of the elastic coupling, will shape the torque vs 
magnitude curves. This maximum torque envelope must be balanced against increases in impedance which affect both safety and performance and represent the basic challenge in implementing the DECMMA approach.

\section{Preliminary Results}

\subsection{Simulation Results: Reduction of Impact Impedance}

The extremely low output impedance of the base actuators and the low impedance of the collocated joint actuators effectively decouple the reflected inertia from the moving parts of the manipulator. This reduction in effective joint inertia is substantial. In the case of a two-axis prototype developed at Stanford, the effective joint inertia was reduced by almost a factor of 10 . We can use the effective inertia, graphically illustrated as a belted ellipsoid [8], to calculate the impulse due to impact at any point on the manipulator. To demonstrate the effectiveness of the DECMMA approach in reducing impact loads, Figure 6 shows the normalized impact impulse for various cases of end-point load for a two degree of freedom planar manipulator. The impact

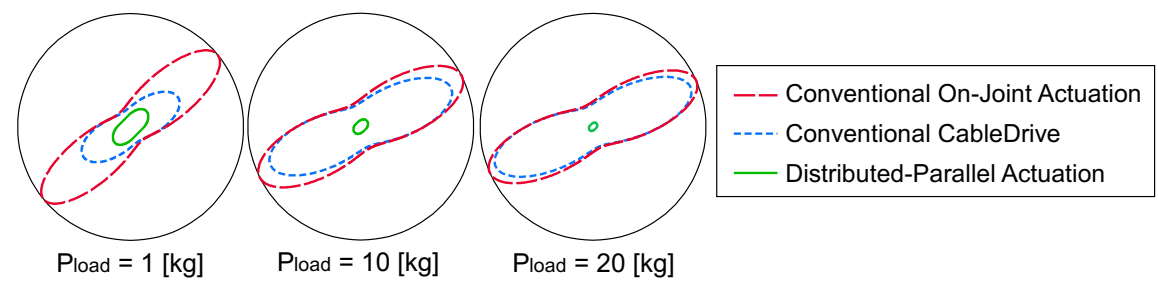

Fig. 6. Comparison of normalized impact impulse for various actuation concepts

impulse reduction increases rapidly with increasing load, as the required increase in actuator torque capability affects the reflected inertia of the conventional and cable-driven manipulators while minimally affecting the reflected inertia of the DECMMA actuators. While this is just a illustrative example, we see that in combination with a light weight structure and compliant covering, the DECMMA approach can be used to design a manipulator that reduces impact loads substantially, thus ensuring inherent safety.

\subsection{Experimental Results}

Preliminary trajectory tracking experiments carried out on a two-axis planar manipulator testbed (see Figure 7) demonstrate the feasibility of the DECMMA approach. Initial experiments demonstrated a position control bandwidth of approximately $5 \mathrm{~Hz}$ as compared to a $2 \mathrm{~Hz}$ bandwidth using the base actuator alone (see Figure 8). Specifically, experiments demonstrated 


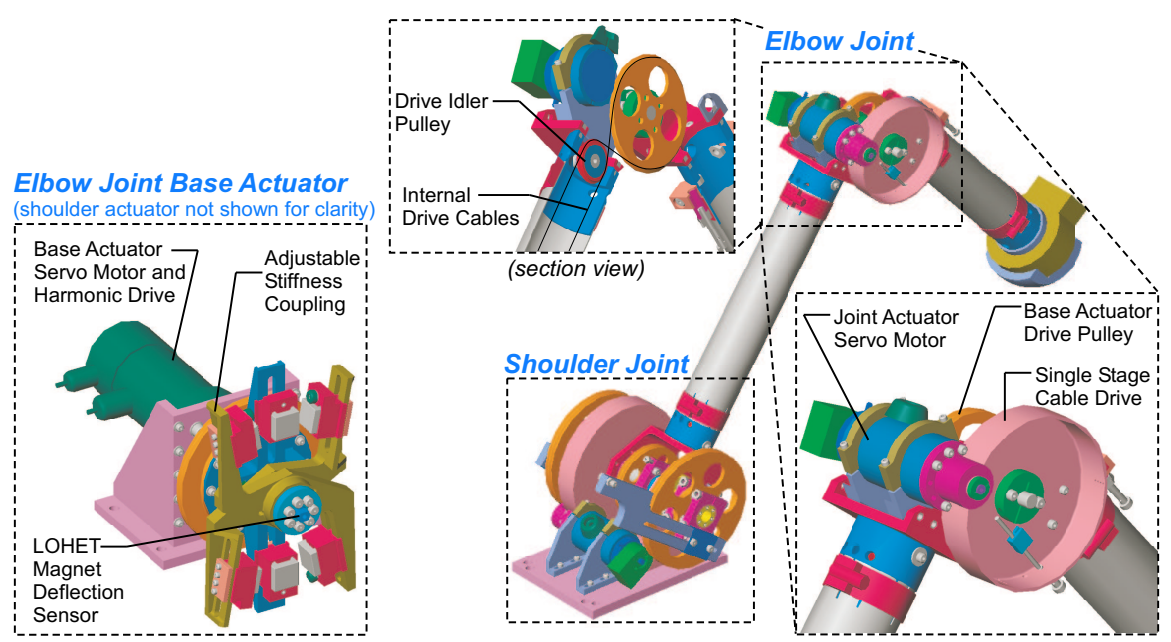

Fig. 7. DECMMA two axis testbed

hardware induced limitations. These include (1) compliance in the joint actuator drive train, (2) saturation of the joint actuator, and (3) structural resonances. The deleterious effect of (3) was by far the most significant factor in limiting the position control performance of the DECMMA testbed. It should be noted that (3) is not a function of the DECMMA concept and doesn't not reflect on its ultimate performance. The effect of (1) and (2) introduce unwanted phase lag into the summed actuation torques. Both (1) and (2) are characteristics of the DECMMA architecture and, as such, require modifications to the existing prototype. We are currently addressing these concerns and expect to have modifications in place in the near future.

Preliminary results, gathered from simple tests and demonstrations, have demonstrated very promising haptic device characteristics, including large work space and large force magnitude capability while maintaining high stiffness characteristics. As with position control, the performance was primarily limited by the structural resonance of the test stand.

\section{Summary}

We have demonstrated a new actuation approach, referred to as Distributed Elastically Coupled Macro Mini Actuation (DECMMA). The new approach substantially reduces the impact loads associated with uncontrolled manipulator collision by relocating the major source of actuation effort from the joint to the base of the manipulator. High frequency torque capability is maintained with the use of small, low inertia servomotors collocated at the joints. The servomotors, integrated with a low reduction, low friction cable transmission, provide the high frequency torque required for high performance tasks while not significantly increasing the combined impedance of 

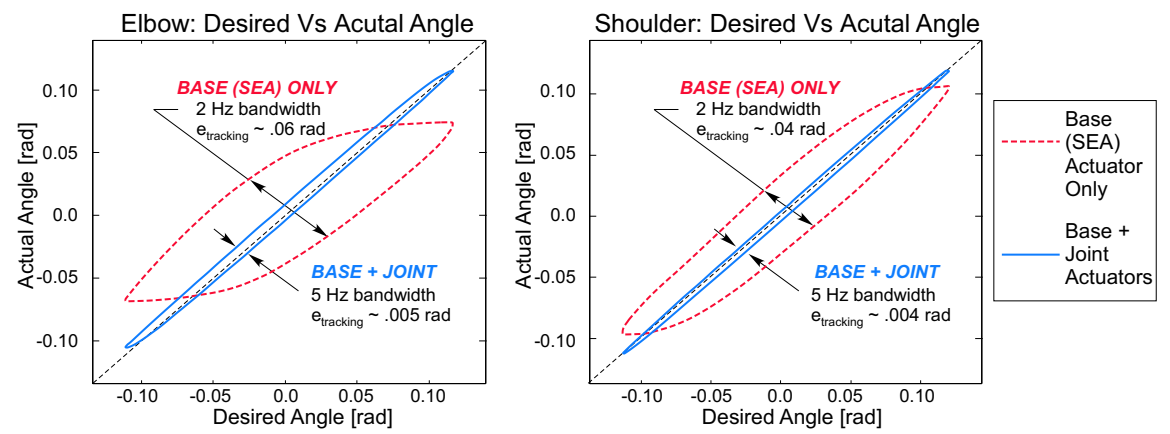

Fig. 8. Comparison of base actuation only (SEA) to combined DECMMA actuation: Position tracking performance

the manipulator-actuator system. The low output impedance and complete frequency coverage of the DECMMA approach allows the combined manipulator system to approximate a pure torque source. This in turn allows for very good open loop joint torque control over a wide frequency range. Initial experimental results in position tracking and haptics validate the DECMMA approach.

\section{Acknowledgments}

The authors would like to thank Gene Duval, Gunter Neimeyer, and Ken Waldron for their helpful insights and discussion in preparing this paper. The financial support of NSF grant EIA-9977717 is gratefully acknowledged.

\section{References}

1. Townsend W. (1988) The Effect of Transmission Design on Force-Controlled Manipulator Performance, Ph.D. Thesis, M.I.T., Cambridge, Massachusetts

2. Holmberg, R. et al (1992) A New Actuation System for High-Performance Torque-Controlled Manipulators, Proc of the 9th CISM-IFToMM Symp Theory and Practice of Robots and Manipulators, Udine Italy, Sept 1992, 285-292

3. Hirzinger G. et al (2001) A New Generation of Torque Controlled Light-weight Robots, Proc of the International Conference on Robotics and Automation

4. Morrel, J.B. (1996) Parallel Coupled Micro-Macro Actuators, Ph.D. Thesis, Massachusetts Institute of Technology, Cambridge, Massachusetts

5. Pratt, G., Williamson, M. (1995) Series Elastic Actuators, Proceedings of the IEEE/RSJ International Conference on Intelligent Robots and Systems, 399-406

6. Robinson, D. (2000) Design and Analysis of Series Elasticity in Closed-loop Actuator Force Control, Ph.D. Thesis, M.I.T., Cambridge, Massachusetts

7. Vischer, D., Khatib, O. (1995) Design and Development of High-Performance Torque-Controlled Joints,IEEE Trans on Robotics and Automation, v11, n4

8. Khatib, O. (1995) Inertial Properties in Robotic Manipulation: An Object-Level Framework, International Journal of Robotics Research, v14, n1, 19-36 


\section{Index}

actuation, 1

human-friendly, 1 impedance, 1

safety, 1 